\title{
Medios de prensa digitales en Chile: influencia del editor y de la audiencia en la reunión de pauta
}

\section{Digital media in Chile: influence of the editor and the audience in the editorial meeting}

\section{Mídia digital no Chile: influência do editor e do público na reunião de pauta}

María Francisca Greene González, Universidad de los Andes, Santiago, Chile (fgreene@uandes.cl)

María José Lecaros Menéndez, Universidad de los Andes, Santiago, Chile (mlecaros@uandes.cl)

María Fernanda Cerda Diez, Universidad de los Andes, Santiago, Chile (mcerda@uandes.cl)

RESUMEN|Esta investigación explora la evolución de la rutina periodística de la pauta en los medios digitales en Chile en los últimos años. Se examina específicamente cómo se elabora la pauta, el rol del editor y la influencia de la audiencia para seleccionar y jerarquizar los contenidos. Se contempló una muestra de los diez medios digitales más leídos en Chile en 2020 y se aplicaron entrevistas en profundidad a sus editores o directores. Los principales resultados apuntan a una diferenciación entre medios matriciales y medios nativos digitales. Mientras que los medios matriciales tienen una injerencia menos directa del editor sobre los periodistas y las respuestas del público afectan fuertemente la selección de contenidos, los medios nativos digitales mantienen un estilo más tradicional de elaboración de sus pautas y noticias. En estos últimos, los editores tienen mayor injerencia sobre los periodistas, las reuniones de pauta suelen hacerse una vez al día y los contenidos están poco condicionados por las métricas y las redes sociales. Aunque el estudio es una fotografía de algunos medios digitales chilenos, permite concluir que la evolución del periodismo en línea en Chile ha sido rápida, se le da mayor importancia a la pauta, se ha perfilado más la figura del editor, y la influencia de la audiencia en los contenidos es cada vez más importante respecto de unos años atrás.

PALABRAS CLAVE: medios digitales; rutinas periodísticas; editor; pauta; audiencia. 
ABSTRACT / The research aims to evaluate how the journalistic routine of the editorial meeting in digital media has evolved in Chile in recent years. Specifically, we examined how the editorial meeting is developed, the role of the editor in it and the influence of the audience to select and rank the contents. We carried out a study of ten cases, which included a sample of the most read digital media in Chile. In-depth interviews were conducted with editors or directors. The main results, in general terms, point to a differentiation between multiplatform matrix media and digital nativemedia. While the former havesubstantially modified their guideline-making routines in recent years, haveless direct interference from the editor over journalists, and public responses strongly affect content selection, native digital media maintain a more traditional style of preparing their guidelines and news. Editors have a greater influence over journalists, editorial meetings tend to be held once a day and content is little conditioned by metrics and social networks. Although the study is a photograph of some Chilean digital media, it allows to conclude that the evolution of online journalism in Chile has been rapid, that greater importance is given to the editorial meeting, that the figure of the editor has been more outlined, and that the influence of the audience's feedback is increasingly important compared to a few years ago.

KEYWORDS: digital media; journalistic routines; editor; editorial meeting; audience.

RESUMO|A pesquisa visa avaliar como a rotina jornalística da pauta na mídia digital tem evoluído no Chile nos últimos anos. Em particular, foi examinado como a pauta é desenvolvida, o papel do editor nela e a influência do público para selecionar e classificar o conteúdo. Foi realizado um estudo de dez casos, que incluiu uma amostra das dez mídias digitais mais lidas no Chile e entrevistas em profundidade foram aplicadas aos editores ou diretores. Os principais resultados apontam para uma diferenciação entre mídia matricial e mídia digital nativa. Embora a mídia matricial tenha menos interferência direta do editor sobre os jornalistas e as respostas do público afetem fortemente a seleção de conteúdo, a mídia digital nativa mantém um estilo mais tradicional criação de pautas e notícias. Neste último aspecto, os editores têm maior interferência sobre os jornalistas, as reuniões de pauta costumam ser realizadas uma vez ao dia e o conteúdo é pouco condicionado por métricas e redes sociais. Embora o estudo seja uma fotografia de alguma mídia digital chilena, permite concluir que a evolução do jornalismo online no Chile foi rápida, maior importância é dada à pauta, a figura do editor foi mais delineada, e a influência da audiência no conteúdo é cada vez mais importante em comparação com alguns anos atrás.

PALAVRAS-CHAVE: mídia digital; rotinas jornalísticas; editor; pauta; audiência. 


\section{INTRODUCCIÓN}

En el ámbito de los estudios del periodismo, la rutina de la pauta en el contexto de los medios digitales ha concitado el interés de distintos investigadores. Agarwal y Barthel (2013) descubren que mientras que los medios digitales se hacen más profesionales, los periodistas redefinen qué significa producir las noticias al tiempo que adaptan rutinas ya existentes del periodismo tradicional.

Los autores que estudian el impacto de lo digital en el periodismo coinciden en afirmar -desde perspectivas diversas- que el fenómeno aún no se ha estabilizado en el mundo (Salaverría, 2016). En este nuevo entorno informativo se privilegia la rapidez por sobre la objetividad (Cassidy, 2005; Schudson \& Anderson, 2009). Para facilitar este flujo de trabajo, los periodistas del medio digital trabajan de manera independiente (Singer, 2004) y hacen su reporteo principalmente en línea (O’Sullivan \& Heinonen, 2008; Salwen, 2005). La producción de los periodistas usualmente no depende de la plataforma, y producen indistintamente noticias en texto, video o multimedia (Mitchelstein \& Boczkowski, 2009; Singer, 2004). La web también ofrece a los periodistas un mayor acceso a las fuentes. Sin embargo, la rutina que más ha cambiado y que es más estudiada es la nueva relación que se establece con la audiencia, en el entendido de que la participación del público condiciona el producto noticioso y las pautas diarias (Tandoc \& Ferrucci, 2017). Interesa estudiar la pauta, por constituir un momento privilegiado del trabajo profesional en el que el "aporte intelectual y la toma de decisiones marca fuertemente la diferencia en el producto final” (Greene-González \& Lecaros-Menéndez, 2015, p. 591).

En el caso de Chile, la rutina periodística de la pauta ha sido estudiada desde distintas perspectivas (Stange \& Salinas, 2009; Puente \& Grassau, 2011; Lecaros \& Greene, 2012; Puente et al., 2014; Greene \& Lecaros, 2015; Greene González, 2016, 2017). Este estudio retoma una investigación iniciada en 2014 en el país sobre la pauta en el periodismo digital chileno. Entre 2014 y 2017, los estudios de Greene González $(2016,2017)$ y Greene-González y Lecaros-Menéndez (2015) dan cuenta de dos fenómenos: la incorporación progresiva de la audiencia en la elaboración de la pauta y cómo, en los medios en línea, la figura tradicional del editor se desperfila respecto del rol que tenía en los medios offline. El estudio de 2015 analiza los tres primeros medios digitales chilenos que apuestan por sumar a la audiencia en su pauta. Uno de los medios incluso habla de pauta compartida. En el trabajo de 2015 se observa en los medios en línea una pauta como rutina heredada de los medios clásicos, no adaptada a la prensa digital, y un trabajo del editor desperfilado frente a una mayor autonomía del periodista. Esta tendencia se mantiene en el estudio de 2016. En el estudio de 2017, la figura del editor aparece como clave para lograr un buen trabajo de pauta y la audiencia no condiciona mayormente el trabajo del periodista. 
Así, el objetivo de este trabajo fue investigar la dinámica de elaboración de la pauta en medios digitales durante 2019 desde la perspectiva de los editores de los medios con mayor audiencia, según los rankings de Alexa (Alexa, 2019) y ComScore (ComScore, 2019), de modo de descubrir qué incidencia tienen en ella el editor y la audiencia. Se intentó descubrir, además, si hay diferencias entre los denominados medios nativos digitales y matriciales (es decir, nacidos al alero de un soporte tradicional) ${ }^{1}$.

\section{El cambio de la pauta en el periodismo digital}

Para entender la evolución de la pauta interesa rescatar las tres etapas de la aparición de lo digital en el periodismo propuestas por Vobič (2015).

En la primera etapa (décadas de 1980/1990), los medios tradicionales exploran y comienzan un intento de apropiación del mundo digital, no solo como nuevo modelo de negocio, sino también desde las decisiones editoriales y la producción de noticias.

La segunda etapa (entre los años 1990 y 2000) está marcada por la incertidumbre sobre qué es lo propio de un diario digital; las interrogantes van "desde el qué hacer, cómo hacerlo, quién lo debe hacer, hasta cómo se evalúa lo hecho” (Vobič, 2015, p. 177).

En la tercera etapa (posterior a 2000) se producen cambios importantes. Los medios tienen ciclos de entrega de 24 horas. Las políticas de decisión se hacen flexibles, se dispersan las decisiones y sobre los periodistas recae una fuerte carga de trabajo. El periodista online enfrenta desafíos variados y las soluciones que se proponen son disímiles (Vobič, 2015). Se elaboran noticias especialmente para

1. Según Tanner (1999) los medios en Internet aparecen en 1994 en Chile, cuando comienzan a publicar parte de sus contenidos en la web: Siglo XXI -suplemento semanal de ciencia y tecnología de El Mercurio- y el diario Financiero y Estrategia. El Grupo COPESA comenzó a publicar resúmenes semanales de noticias y editoriales de La Tercera, siendo el primer periódico chileno en Internet (junio 1996). Sin embargo, cita al desaparecido La Época como el primer periódico chileno en publicar información diaria en la web. Los medios en línea chilenos han mostrado entusiasmo en adoptar las redes sociales. Si bien los estudios disponibles no registran los primeros en utilizar Facebook, Jofré (2017) sugiere que dos periódicos del grupo COPESA, La Tercera y La Cuarta, fueron pioneros en usar Twitter (abril de 2007); radio Cooperativa (julio 2007) y La Nación (noviembre 2017). A partir de 2000, los medios digitales se multiplican, siendo El Mostrador uno de los primeros medios nativos digitales. Para 2020 todos los principales medios nacionales tienen formato en línea. Incluso medios como La Tercera deciden limitar las impresiones del formato en papel solo para el fin de semana, priorizando el contenido en línea. 
la versión web (Pavlik, 2008). Henkel y sus colegas (2020) concluyen que, en los últimos años, aumenta la calidad del periodismo en línea y que las rutinas de los periodistas de medios digitales son más similares a las de los periodistas que lo hacen en medios offline que hace algunos años.

\section{Características de las noticias en la pauta digital}

Pareciera que la tercera etapa está impulsada por la velocidad, la inmediatez, la hipertextualidad, la interactividad y la multimedialidad propias del mundo digital (Vobič, 2015). La velocidad es la que empuja el trabajo al preparar las ediciones en línea (Fortunati \& Sarrica, 2011). Para responder a la inmediatez, los periodistas casi nunca proveen de contenidos originales; optan por hacer un "periodismo de segunda mano" (Quandt, 2008, p. 723) y "monitorean a otros medios" (Boczkowski, 2009, p. 101).

Agarwal y Barthel (2013) concluyen que las ideas para la noticia -momento clave de la pauta-generalmente provienen del interés del periodista, del suministro de las redes sociales o de conversaciones con colegas. Los editores habitualmente no sugieren noticias: trabajan a partir de conversaciones tanto online como offline. Las reuniones de pauta con todo el equipo son cada vez menos comunes para los periodistas online. A cambio, están en contacto directo con el editor vía email o WhatsApp (Lee \& Tandoc, 2017). La reunión de pauta es reemplazada por una reunión virtual y continua con el editor, facilitada por el teléfono móvil. Concluyen que las normas tradicionales se están debilitando, pero que al adaptanrse a las condiciones del trabajo periodístico en línea no desaparecen.

\section{El editor y la pauta de los medios digitales}

Para los editores chilenos, la pauta es considerada como la primera de las rutinas. Es el momento de la jornada al que se le otorga mayor importancia (Lecaros \& Greene, 2012; De Fontcuberta, 1999), cuando se toman decisiones que afectan a los ciudadanos y a las instituciones sociales (Pellegrini, 1989). Permite entender el rol que juegan los periodistas y las relaciones que establecen con su editor; es una rutina que se da en un ambiente físico y social (Quandt, 2008), por tanto, fácilmente observable.

Tradicionalmente se priorizaba la autonomía del editor. Las teorías acerca de la construcción de las noticias han estudiado e identificado influencias potenciales en los editores, asumiendo que los periodistas protegen su trabajo de algunas presiones como las del gobierno, las fuentes y la audiencia (Shoemaker \& Vos, 2009). Agarwal y Barthel (2013) sostienen que los medios habitualmente se basaban en un sistema jerárquico. Se relaciona habitualmente el rol del editor en la pauta con seleccionar y jerarquizar las noticias más relevantes para la toma de decisiones de los ciudadanos (Stange \& Salinas, 2015; Puente et al., 2014). Sin embargo, estudios 
recientes han descubierto que pareciera que la figura del editor en los medios digitales tiene menos poder de decisión frente a los periodistas que conforman su equipo en comparación con la figura del editor del diario en papel (Greene \& Lecaros, 2015; Greene González, 2016) y que esta relación es más horizontal que en los medios tradicionales (Gronemeyer, 2013). Finalmente, $\mathrm{Vu}$ (2014) concluye que los editores con menos años de experiencia tienden a dejarse influir más por las métricas en sus criterios de selección de las noticias, lo que introduce la variable del rol de la audiencia en la pauta.

\section{El rol de la audiencia en el armado de la noticia}

Probablemente el cambio más fundamental en las decisiones editoriales proviene de la inclusión de la audiencia en la pauta. Según Doval-Avendaño y Algarra (2021), uno de los grandes cambios en el periodismo producto de las innovaciones tecnológicas es la relación existente entre los medios y la audiencia. Mientras que en la era predigital el concepto de la audiencia que tenían los periodistas era una "ficción operativa" (Zurstiege, 2006, p. 72) las métricas alteran su trabajo en las decisiones noticiosas. ¿Cuáles son las consecuencias de una audiencia más conocida en las decisiones editoriales? Las investigaciones no son concluyentes y muestran resultados dispares. MacGregor (2007) afirma que los clics llevan a los periodistas a profundizar en la noticia y a publicar noticias del mismo tipo. Boczkowski y Peer (2011) concluyen que la apuesta noticiosa de los periodistas y la de la audiencia no siempre coinciden.

Tandoc y Ferrucci (2017) citan dos insumos de la audiencia como relevantes en este nuevo escenario: las redes sociales y la web analytics. Los autores plantean hasta qué punto afectan el trabajo periodístico tradicional y cómo inciden en la autonomía de la toma de decisiones, así como en los valores clásicos de la profesión.

El público no solo aporta una enorme cantidad de información, sino que tiene la capacidad de ingresarla en las distintas etapas del ciclo noticioso e impactar en cada una de ellas (Tandoc \& Ferrucci, 2017). Según Hanusch (2017), los periodistas son cada vez más conscientes de la importancia/interés de estos datos: los consultan muchas veces cada día y tienen una percepción positiva de este insumo. La data les permite saber quién accede, a qué notas, a qué hora, dónde y cuánto tiempo destina a la lectura. La métrica les permite experimentar en la selección de imágenes y contenidos, entre otras acciones. No obstante, según el autor, este mayor conocimiento de la audiencia puede significar una pérdida de la autonomía y capacidad de decisión del profesional.

Estos cambios fortalecen el periodismo participativo, destacando la naturaleza colaborativa y colectiva en la producción de las noticias que se facilita con las secciones de comentarios, foros, redes sociales, etc. (Singer et al., 2011), donde 
también se puede interactuar con otros usuarios (Bucy, 2004). Esa participación, sin embargo, no solo permite la comunicación entre quienes están dentro y afuera de la sala de redacción, sino que afecta también lo que llegará a ser noticia. Asimismo, facilita la comunicación entre quienes están fuera de la sala de redacción e incide en cómo la noticia es seleccionada, procesada e interpretada (Lee \& Tandoc, 2017).

La digitalización de las noticias ha dado origen a nuevas formas de retroalimentación por parte de la audiencia. Los comentarios publicados son visibles instantáneamente para otros lectores (Craft et al., 2016). Las redes sociales como Twitter y Facebook también proveen de listas de trending topics. Los algoritmos rastrean lo que el público comenta y reenvía, junto con data en tiempo real a través de likes, comentarios y compartidos de un post (Hermida, 2011; Tandoc \& Vos, 2016).

Comparado con los sistemas tradicionales, estas nuevas formas de retroalimentación de la audiencia son más rápidas, automáticas e inclusivas, pues provienen de un mayor número de personas que antes; también son más completas, al incluir formas textuales y numéricas, y más públicas, ya que son visibles no solo para el medio sino también para el resto de los usuarios (Lee \& Tandoc, 2017).

Las reuniones de pauta suelen comenzar con la discusión de los trending topics en las redes sociales, y los editores determinan los eventos, temas o tópicos en los que las audiencias digitales pueden estar interesadas (Tandoc \& Vos, 2016; Welbers et al., 2016). Estas nuevas formas de retroalimentación de la audiencia acrecientan su influencia en la producción de noticias, desafiando a las teorías clásicas del gatekeeping y de la agenda setting (Chaffee \& Metzger, 2001; Shoemaker \& Vos, 2009; Duffy, 2019). Por ejemplo, los estudios citan "mayor compromiso con las audiencias en el proceso de selección de las noticias" (Lee et al., 2014, p. 505), lo que hace que la agenda setting en línea sea un "proceso recíproco" en lugar de ser originado solo desde los medios (Ragas et al., 2014, p. 57).

Otros autores sostienen que la retroalimentación de la audiencia puede implicar que el lector olvide la postura editorial del medio (Lee \& Ma, 2012; Gunther \& Liebhart, 2006) y también puede alterar la percepción de la calidad de una noticia (Weber, 2014).

Los periodistas, por una parte, están desafiados a desarrollar rutinas para usar estas métricas, principalmente debido a la influencia de la lógica de mercado (Gans, 1980; Hamilton, 2004; Vu, 2014). Por otra parte, están constreñidos por las normas tradicionales del periodismo (Anderson, 2011). Domingo (2008), por ejemplo, sostiene que hay un mito acerca de la interactividad en el periodismo digital, forjado en las mentes y los discursos de los periodistas digitales. 
Para ellos, la interactividad con la audiencia constituye más un problema que una oportunidad para el cambio. La cultura profesional del periodismo tradicional juega un rol más importante (y seguramente inconsciente), como se demuestra en la diversidad de estrategias de los medios que son solo digitales de la de aquellos que están asociados a medios tradicionales (Domingo, 2008). Lee y sus colegas (2014) sostienen que, mientras que los periodistas de medios en línea usan las métricas para tomar decisiones estratégicas, los editores evitan seguir servilmente los clics. Junto con los cambios permanece una preservación subyacente de los ideales profesionales (Singer, 2011). En consecuencia, los periodistas luchan por lograr un equilibrio entre el uso de las métricas con las normas tradicionales (Anderson, 2011; Lee et al., 2014; Lewis \& Usher, 2013). Tanto los estudios de Lee y sus colegas (2014) como los de Welbers y sus colaboradores (2015) concluyen que a los editores les cuesta admitir su dependencia de las métricas.

En ese contexto, los objetivos de esta investigación buscan caracterizar el rol del editor y de la audiencia en la elaboración de la pauta y establecer similitudes y diferencias entre los medios nativos digitales y matriciales.

\section{METOdOLOGÍA}

Se condujo una revisión bibliográfica sobre las características de la pauta en los medios de comunicación digitales y se realizaron entrevistas en profundidad a diez periodistas chilenos que actualmente se desempeñan como editores o directores en medios digitales, con firma del debido consentimiento informado por cada participante.

Se seleccionaron los diez medios digitales chilenos más vistos según los rankings de Alexa (Alexa, 2019) y ComScore (ComScore, 2019): emol.com, latercera.com, biobio.cl, cooperativa.cl, 24horas.cl, df.cl, elmostrador.cl, ellibero.cl, eldinamo. cl y pauta.cl (tabla 1). Cinco son medios nativos digitales (emol.com, ellibero.cl, pauta.cl, elmostrador.cl y eldinamo.cl) y cinco son medios matriciales (latercera. com, biobio.cl, cooperativa.cl, 24horas.cl y df.cl).

En la selección de los diez casos se utilizó el muestreo cualitativo por conveniencia, buscando la representatividad del universo de medios digitales chilenos (Navarrete, 2000). Estos casos se consideran unidades teóricas de un mismo fenómeno, similar a la lógica de la replicación pura (Eisenhardt, 1989; Yin 2009; Swanborn, 2010, p. 104).

Para las entrevistas en profundidad se seleccionaron directores y editores. Los entrevistados utilizan distintos términos para designar funciones similares. 


\begin{tabular}{|c|c|c|c|c|c|}
\hline Medio & Plataforma & $\begin{array}{c}\text { Cargo del } \\
\text { entrevistado }\end{array}$ & $\begin{array}{l}\text { Año de } \\
\text { creación }\end{array}$ & $\begin{array}{l}\text { Sociedad } \\
\text { comercial }\end{array}$ & Objetivos del medio \\
\hline EMOL & Nativo digital & $\begin{array}{l}\text { Director de } \\
\text { EMOL }\end{array}$ & 1990 & $\begin{array}{l}\text { El Mercurio } \\
\text { S.A.P. }\end{array}$ & $\begin{array}{c}\text { Informar con rapidez y } \\
\text { objetividad a. }\end{array}$ \\
\hline $\begin{array}{l}\text { La Tercera } \\
\text { Online }\end{array}$ & Matricial & $\begin{array}{l}\text { Subeditor de } \\
\text { La Tercera en } \\
\text { Internet }\end{array}$ & 1997 & Grupo Copesa & $\begin{array}{c}\text { Informar, interpretar y } \\
\text { contextualizar las } 24 \text { horas } \\
\text { a través de las distintas } \\
\text { plataformas y formatos. } \\
\text { Poner el periodismo al servicio } \\
\text { de los usuarios y sus variados } \\
\text { intereses }{ }^{b} \text {. }\end{array}$ \\
\hline BioBio Chile & Matricial & $\begin{array}{l}\text { Editor } \\
\text { contenidos } \\
\text { digitales }\end{array}$ & 2009 & $\begin{array}{c}\text { Biobío } \\
\text { Comunicaciones }\end{array}$ & $\begin{array}{c}\text { Informar con veracidad y } \\
\text { objetividad sobre el acontecer } \\
\text { nacional }{ }^{c} \text {. }\end{array}$ \\
\hline $\begin{array}{c}\text { Radio } \\
\text { Cooperativa } \\
\text { online }\end{array}$ & Matricial & $\begin{array}{l}\text { Director } \\
\text { de Radio } \\
\text { Cooperativa } \\
\text { y medios } \\
\text { digitales }\end{array}$ & 2001 & $\begin{array}{c}\text { Compañía } \\
\text { Chilena de } \\
\text { Comunicaciones } \\
\text { S.A. }\end{array}$ & $\begin{array}{c}\text { Informar con objetividad, } \\
\text { imparcialidad y seriedad las } \\
\text { informaciones provenientes } \\
\text { de todos los sectores de la vida } \\
\text { nacional e internacional }{ }^{\mathrm{d}} \text {. }\end{array}$ \\
\hline $\begin{array}{c}\text { Diario } \\
\text { Financiero }\end{array}$ & Matricial & Director & 2018 & $\begin{array}{c}\text { Ediciones } \\
\text { Financieras S. A. }\end{array}$ & $\begin{array}{l}\text { Informar y dar un contexto } \\
\text { sobre las noticias económicas } \\
\text { para permitir a los lectores } \\
\text { tomar decisiones informadas }\end{array}$ \\
\hline TVN Online & Matricial & $\begin{array}{l}\text { Editor general } \\
\text { plataformas } \\
\text { digitales TVN - } \\
24 \text { Horas }\end{array}$ & 2001 & $\begin{array}{c}\text { Televisión } \\
\text { Nacional de Chile }\end{array}$ & $\begin{array}{l}\text { A través de la producción de } \\
\text { información, integrar a toda } \\
\text { la población a la vida social, } \\
\text { cultural y política del país }{ }^{f} \text {. }\end{array}$ \\
\hline El Mostrador & Nativo digital & $\begin{array}{c}\text { Editor } \\
\text { periodístico }\end{array}$ & 2000 & Plaza S.A. & $\begin{array}{l}\text { Informar con independencia } \\
\text { y pluralismo, respetando y } \\
\text { valorando la diversidad y los } \\
\text { derechos humanos y civiles. } \\
\text { Fiscalizar los poderes } \\
\quad \text { establecidos. } \\
\text { Dialogar con la ciudadaníag. }\end{array}$ \\
\hline El Dínamo & Nativo digital & $\begin{array}{l}\text { Editor en jefe- } \\
\text { Director }\end{array}$ & 2010 & Giro País SPA & $\begin{array}{c}\text { Informar en forma veraz y con } \\
\text { altos estándares de calidad a su } \\
\text { audiencia }{ }^{h} \text {. }\end{array}$ \\
\hline El Líbero & Nativo digital & Director & 2014 & $\begin{array}{l}\text { Sociedad } \\
\text { Periodística El } \\
\text { Líbero S.A. }\end{array}$ & $\begin{array}{l}\text { Ayudar a satisfacer las } \\
\text { necesidades informativas } \\
\text { de la audiencia entregando } \\
\text { una mirada editorial propia, } \\
\text { inspirada en la libertad, } \\
\text { que sirva de alternativa o } \\
\text { complemento a los medios } \\
\text { existentes en la industria }\end{array}$ \\
\hline Pauta & Matricial & Editor general & 2018 & Voz Cámara SpA & $\begin{array}{l}\text { Proporcionar información } \\
\text { relevante y pluralista a sus } \\
\text { audiencias para que puedan } \\
\text { tomar decisiones que influyan } \\
\text { positivamente en la sociedad }{ }^{j} \text {. }\end{array}$ \\
\hline
\end{tabular}

Nota. ${ }^{\mathrm{E} E m o l}$ (2019). ${ }^{\mathrm{b}}$ La Tercera (2020). ${ }^{\mathrm{C}}$ Radio BioBio Chile (2016). ${ }^{\mathrm{d}}$ Cooperativa.cl (2021). ${ }^{\circ}$ Diario Financiero

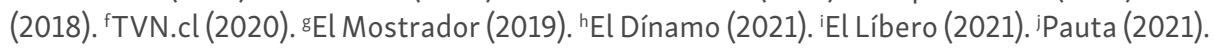

Tabla 1. Medios de comunicación consultados

Fuente: Elaboración propia. 
Si bien en algunos medios se entrevistó al editor general, en otros pareció relevante entrevistar al director, ya que algunos ejecutan ciertas labores propias del editor.

Las entrevistas en profundidad buscan entender "la experiencia, el conocimiento y las visiones del mundo del actor social" (Lindlof \& Taylor, 2011, p. 173). Mediante preguntas semiestructuradas y una discusión temática, se logra una comprensión más rica del grupo estudiado (Tracy, 2019).

Previo consentimiento oral o escrito, las entrevistas se desarrollaron entre octubre de 2019 y marzo de 2020 y duraron entre 20 a 40 minutos. Cinco se realizaron antes del 18 de octubre de 2019. En esta fecha, en Chile, se inició un movimiento social de envergadura, sobre todo en la capital (lugar de las entrevistas), lo que llevó a restringir algunas de las libertades individuales (toque de queda, entre otras medidas) y prácticamente dominó la agenda de los medios. Un escenario similar de confinamiento se vivió al inicio de la pandemia de COVID-19, por lo que podría esperarse una modificación de sus rutinas. Sin embargo, los entrevistados afirmaron explícitamente que las rutinas relacionadas con la reunión de pauta, sus roles como editores y la incorporación de la audiencia en la toma de decisiones no sufrieron cambios con este acontecimiento. Las primeras entrevistas se hicieron en formato presencial y luego del 18 de octubre de 2019, mediante llamadas telefónicas o Zoom. Estas fueron grabadas o se tomaron notas durante las llamadas y luego fueron transcritas en su totalidad.

Los temas trataron sobre la definición de la pauta, el trabajo del editor y el protagonismo de la audiencia.

\section{Análisis de contenido de las entrevistas}

El análisis de contenido de las entrevistas se basa en la lectura (textual o visual) siguiendo el método científico, lo que aporta una estructura objetiva, replicable y válida (Andréu Abela, 2001).

De las transcripciones de las entrevistas a editores y directores de medios digitales se extrajeron las unidades de análisis, que surgieron como un grupo de palabras, oraciones o párrafos de las entrevistas transcritas (Krippendorff, 2004). Se utilizó codificación emergente e inductiva para analizar los datos, a cargo de dos investigadores, quienes asignaron códigos a los datos brutos. Se creó un libro de códigos emergentes, al que los investigadores se refirieron regularmente en todas las fases del análisis. A medida que surgían nuevos códigos, se revisaban los textos previamente codificados y, si era necesario, se recodificaban. En el segundo nivel de codificación, los investigadores identificaron similitudes y patrones entre los códigos, que luego fueron categorizados en temas (Saldaña, 2012). Mediante este proceso surgieron un total de cuatro temas con 16 códigos (tabla 2). 
Tema

Pauta

Tipos de medio
Código

Fuentes

Alertas noticiosas

Portada

Contenido

Retroalimentación del público

Redes sociales (Twitter, WhatsApp)

Métrica

Comentarios

\section{Cierre}

Línea editorial

Competencia

Evolución del medio

Periodista

Trabajo en equipo

Editor

Edición

Rutina

\section{Tabla 2. Categorización temática}

Fuente: Elaboración propia.

\section{RESULTADOS}

Los resultados obtenidos se clasifican en tres grandes temas: (i) tipos de medios en línea y contenidos de cada uno de ellos, (ii) rol del editor y elaboración de la pauta, y (iii) retroalimentación de la audiencia, su influencia y su interacción con la línea editorial.

\section{Rol de editor en la elaboración de la pauta}

El rol del editor de los medios nativos digitales es más parecido al de un medio tradicional. Los editores de medios nativos digitales suelen ser más senior y todos han sido editores de un medio tradicional escrito.

Como las noticias que suben son menos numerosas, revisan todas las notas, con títulos y bajadas. Los editores dan el foco (ellibero.cl), aseguran la línea editorial del medio (ellibero.cl), constituyen un "filtro más afinado" (pauta.cl), en donde "nadie publica solo" (pauta.cl) y “dan los criterios de selección en cuanto a la importancia de un tema" (pauta.cl). "Esa es mi pega ${ }^{2}$ principal, llevar el pulso del diario político y tratar de ir un paso adelante" (elmostrador.cl). Los editores suelen estar presentes en todo el proceso de elaboración y jerarquización de lo que se sube a la web. Los temas se programan más a largo plazo y deciden también qué tema se sube qué día. Estos resultados son consistentes con las funciones del editor descritas por Agarwal y Barthel (2013), Gans (2003), Stange y Salinas (2015) y Puente y sus colegas (2014).

2. Chilenismo para la palabra trabajo. 
Por el tipo de contenidos que publica, emol.com es una excepción respecto de los otros medios nativos digitales, en cuanto a la elaboración de la pauta y a la incidencia del editor en esta. Se hacen varias pautas al día, la relación entre el editor y los periodistas es menos estrecha. En este sentido, funciona como un medio matricial.

Los medios matriciales tienen más diversificada la labor de edición, resultados consistentes con las funciones de editor que establecen Gronemeyer (2013), Greene y Lecaros (2015), Greene González (2016), Boczkowski y De Santos (2007), Cassidy (2005), Klinenberg (2005) y Quandt (2008).

Todos estos medios tienen un editor de mañana y otro de tarde, además de editores de área, subeditores y coordinadores. Los periodistas ven sus notas con el editor de área: solo llegan al editor general las notas más importantes. Los editores reconocen que los periodistas tienen una mayor autonomía para generar contenidos que en los medios tradicionales.

"El editor es un punto de referencia ante dudas" (biobio.cl). "El trabajo del editor es de coordinador general de las tareas del equipo, otorga prioridades, enfoques. Es el nexo entre la edición del medio tradicional y el equipo de prensa web. También monitorea el trabajo de otros medios para ver que no se les escape nada" (biobio.cl).

Ambos modelos de medios digitales tienen semejanzas en cuanto al trabajo del editor: ellos toman las decisiones sobre los cambios de portada.

Los medios nativos digitales elaboran la pauta de modo semejante a como lo hace el diario en papel. Los editores identifican una pauta diaria y una semanal. En los medios nativos digitales hay dos pautas paralelas: la que se trabaja semanalmente, con temas que se reportearán en profundidad, y la pauta diaria, que suele ser solo una vez al día.

Como el flujo noticioso es tan alto, en los medios matriciales, habitualmente hay dos pautas al día: mañana y tarde. Estos editores también tienen su propia pauta con los editores de las otras plataformas. Para ellos, es importante el trabajo que los periodistas del soporte offline hacen para la versión digital. Las reuniones suelen ser con diferentes grupos: editor general con editores de área, y estos con periodistas.

La pauta de medio día suele ser una reunión para ver temas de la tarde y evaluar la primera parte de la jornada. "Planificamos lo que hay que hacer según consideraciones editoriales, vemos qué ha pasado el día anterior, vemos las métricas, qué ha funcionado, qué no, dónde vamos a estar enfocados" (TVN.cl). "Hay que dejar un pedazo de la pauta libre para reaccionar bien ante el último minuto" (TVN. cl). En este sentido, se trata de una pauta que se va ajustando: "si algo se sube, se bajan cosas según los requerimientos de lo que va pasando” (latercera.com). 


\section{Relación con la audiencia y métricas}

Los entrevistados de medios nativos digitales dicen que las métricas "no afectan la pauta" (ellibero.cl). "Antes las bajas de tráfico significaban un cambio en la pauta, no tenemos por qué trabajar en función del tráfico. La falta de visitas se supera de otra manera: creando secciones o contenidos que afirmen el tráfico" (eldinamo.cl). "Hay otras maneras de reafirmar la identidad" (eldinamo.cl). Esta mirada está en concordancia con los hallazgos de Domingo (2008), Lee y sus colegas (2014) y Singer (2011).

La relación con las métricas de los medios matriciales es muy diferente. Estas reflejan los hallazgos de Hanusch (2017), Tandoc y Ferrucci (2017), Singer y sus colegas (2011), Lee y Tandoc (2017) y Craft y colaboradores (2016). "Es clave el feedback del público, trabajar en sintonía con las audiencias, escuchar más la demanda" (cooperativa.cl). "Nos condiciona, tenemos una aplicación que ve qué se está leyendo más en el sitio" (latercera.com). “Tenemos la suscripción digital en el centro de nuestro trabajo. Entregar a los lectores la información que ellos necesitan significa conocerlos mucho. Al suscriptor en papel no lo conocemos, en cambio, al suscriptor digital, lo seguimos. Eso significa que nuestras decisiones están influenciadas sobre todo por lo que el lector necesita" (df.cl). Los editores usan una serie de métricas. "En la reunión de pauta de la mañana revisamos indicadores: qué y cuánto han leído nuestros lectores digitales el día anterior. Las notas que se leyeron hasta el final se priorizan por sobre las que han tenido una lectoría corta" (df.cl). "También tenemos indicadores de visitantes únicos, de visitantes totales. Eso nos permite tomar decisiones" (df.cl). De esta manera aseguran que, "la data en la sala de prensa, bien usada, es muy positiva" (Diario Financiero). Estos resultados son coherentes con las investigaciones de Welbers y sus colegas (2016) y Ragas y sus colaboradores (2014).

"Miramos cuántos clics hemos tenido en cada noticia, las tendencias de Google y cómo está el match entre lo que nosotros pensamos que es importante y lo que al público le interesa" (cooperativa.cl). "La métrica es nuestra Biblia. Nos dice de las cosas que estamos haciendo, dónde está el interés de las personas. Trabajamos con Google Analytics abierto. Las métricas son nuestra hoja de ruta diaria, semanal, mensual; primero, para mantener buenos números y segundo, para entender lo que está buscando la gente en digital" (24horas.cl).

La relación con la audiencia también la obtienen a través de las redes sociales, especialmente de Twitter. Para los medios matriciales, la interacción con la audiencia mediante las redes sociales es clave. "Cada periodista tiene que saber redactar para las redes sociales. Las hemos integrado al trabajo. Twitter es más fácil porque se pueden transmitir noticias duras. Nos interesa habilitar el WhatsApp por el insumo 
de lo que dice la audiencia" (cooperativa.cl). "Las redes sociales son nuestro caballito de batalla. La gente está en las redes sociales y ahí es donde nosotros tenemos que entrar a competir con las otras marcas y los otros medios" (24horas.cl).

De las entrevistas surge un tema interesante: la relación entre la línea editorial de los medios y el aporte de la audiencia. En los medios matriciales, los entrevistados declaran que hacen compatible la línea editorial con los intereses de la audiencia. "Hay un equilibrio entre lo que el medio considera importante y la línea editorial del medio con lo que le interesa a la gente" (cooperativa.cl). "Hay una parte que tiene que ver con los indicadores y una parte que tiene que ver con la línea editorial. Hay cosas que nos pueden parecer súper atractivas, pero no las usamos porque no nos interesan" (df.cl). En los medios nativos digitales, la línea editorial es más autónoma. "Hay una cultura periodística propia del medio, mirada alternativa, de nicho" (ellibero.cl).

\section{Estructura y contenidos: semejanzas y diferencias}

De las entrevistas surgieron semejanzas y diferencias importantes que distinguen medios matriciales de nativos digitales. En relación con la estructura, los primeros trabajan con periodistas enfocados en producir solo contenidos digitales, mientras otro grupo produce contenidos para las plataformas de papel, radio, televisión y, además, contenidos digitales. Disponen de un equipo promedio de 40 periodistas. En ellos se privilegia el trabajo en equipo entre la plataforma digital y la tradicional (los periodistas que trabajan en la radio, televisión o en la versión en papel). Todos los entrevistados coinciden en que los periodistas del medio tradicional trabajan también para la versión en línea.

En los medios nativos digitales trabajan, en promedio, 20 o menos periodistas.

En esta diferenciación entre medios matriciales y nativos digitales hay una excepción: el modelo que utiliza emol.com, que funciona como matricial, pero, a la vez, como nativo digital. En cuanto a contenidos, privilegia la contingencia, el cambio rápido de portada, las noticias en desarrollo, pero con "escaso análisis" (emol.com).

En materia de contenido, los medios nativos digitales lo califican de "poco y bueno, serio" (pauta.cl), y privilegian el reporteo en profundidad antes que subir una nueva noticia. Están menos influidos por el "golpe noticioso". Funcionan más como una "revista diaria" (ellibero.cl). Los fines de semana publican contenido "de consumo lento, pero interesante" (pauta.cl). La portada se cambia, en promedio, unas cuatro a cinco veces al día, mientras que, en los otros, unas catorce veces. Los medios nativos digitales dan "segundas miradas" a los temas, buscan profundizar las breaking news (pauta.cl). Los editores coinciden en que se privilegia la calidad de la información versus la cantidad. Hablan de "contenidos propios", "de largo plazo", 
de una "jerarquía de temas" y, en este sentido, "se sienten" más parecidos a los medios tradicionales. Tienen reportajes que preparan durante la semana y columnas de opinión (elmostrador.cl). Dicen que intentan hacer un "periodismo de interpretación" (elmostrador.cl). Privilegian los temas originales, que no son el "recocido" de otros diarios. "Trabajamos bajo la premisa de que no tenemos espacio para publicar cosas que no tienen importancia" (eldinamo.cl).

La realidad de los medios matriciales es diferente. "Se trata mucho de probar, de buscar nuevos nichos, nuevas audiencias" (24horas.cl). Distinguen claramente una edición de la mañana y una de la tarde. Algunos hacen en sus contenidos un "equilibrio entre pauta de análisis y contingencia" (biobio.cl). Dicen que el valor del diario es el cambio de portada (unas 14 veces al día) (cooperativa.cl). La idea es que lo nuevo esté en la portada y que se pueda encontrar la información fresca fácilmente (24horas.cl), para que cada vez que el usuario actualice el sitio encuentre información reciente (emol.com).

\section{CONCLUSIONES}

El periodismo en línea chileno pareciera estar en la tercera etapa que define Vobič (2015). Los medios digitales chilenos tienen ahora ciclos de entrega de 24 horas. Las políticas de decisión son más flexibles, se dispersan las decisiones y sobre los periodistas recae una fuerte carga de trabajo. El periodista online enfrenta nuevos desafíos. Comparando los resultados de las investigaciones anteriores se puede concluir que el periodismo digital nacional ha evolucionado con rapidez.

Las principales diferencias respecto de la fotografía de 2015 se dan en la pauta y en la figura del editor. Si entonces la pauta de los medios digitales era difusa, prácticamente no existía como momento definido, se privilegiaban las conversaciones entre el periodista y el editor, en 2020 todos los medios digitales estudiados tienen bien identificados sus momentos de reuniones de pauta, han solucionado el tema del flujo infinito de noticias estableciendo más momentos de reuniones a lo largo del día y de la semana. La figura del editor ha ido cobrando más fuerza. Con diferentes matices entre los diarios investigados, el editor suele revisar notas, dar foco y ser un referente para los periodistas que trabajan en el medio. Los contenidos del medio en línea se han enriquecido y profundizado respecto de los estudios anteriores. Ya no se trata de periodistas que suben noticias de agencias o hacen recocidos de otros medios sin salir a reportear a la calle. Las declaraciones de los editores respecto de la audiencia son diferentes y se expresan con radicalidad. Los de medios nativos digitales dicen que no afectan la pauta y se muestran indiferentes ante las bajas de tráfico. Los editores de los matriciales, en cambio, declaran que es clave trabajar con las audiencias y que sus decisiones 
están influenciadas por los lectores. Le dan enorme importancia a la métrica y valoran los contenidos que reciben a través de las redes sociales.

El periodismo digital chileno ha tomado dos rumbos distintos producto de su origen. El nativo digital apuesta por la profundidad, relevancia y pocos cambios en los contenidos; el matricial, por dar más noticias y tener al público informado con la mayor rapidez posible. Sin embargo, se trata de una situación en desarrollo.

\section{REFERENCIAS}

Agarwal, S. D. \& Barthel, M. L. (2013). The friendly barbarians: Professional norms and work routines of online journalists in the United States. Journalism, 16(3), 376-391.

https://doi.org/10.1177/1464884913511565

Alexa (2019). Top Sites in Chile. Alexa.com https://www.alexa.com/topsites/countries/CL

Anderson, C. W. (2011, October 26). Notes towards an analysis of computational journalism. HIIG Discussion Paper Series, 2012-1. https://doi.org/10.2139/ssrn.2009292

Andréu Abela, J. (2002). Las técnicas de análisis de contenido: una revisión actualizada (Content Analysis Techniques: An Updated Review). Fundación Centro de Estudios Andaluces.

BioBioChile. (2018). Especial 50 años (50-years special issue). BioBioChile.cl. https://www.biobiochile.cl/especial/50a\%C3\%Blos/

Boczkowski, P. (2009) Rethinking Hard and Soft News Production: From Common Ground to Divergent Paths. Journal of Communication, 59(1), 98-116.

https://doi.org/10.1111/j.1460-2466.2008.01406.x

Boczkowski, P. \& Peer, L. (2011). The choice gap: The divergent online news preferences of journalists and consumers. Journal of Communication, 61(5), 857-876. https://doi.org/10.1111/j.1460-2466.2011.01582.x

Boczkowski, P. \& Santos, M. (2007). When More Media Equals Less News: Patterns of Content Homogenization in Argentina's Leading Print and Online Newspapers. Political Communication, 24(2), 167-180. https://doi.org/10.1080/10584600701313025

Bucy, E. P. (2004). The interactivity paradox: closer to the news but confused. In E. P. Bucy \& J. E. Newhagen (Eds.), Media access: Social and psychological dim $1^{*}$ ensions of new technology use (pp. 47-72). Erlbaum.

Cassidy, W. P. (2005). Variations on a Theme: The Professional Role Conceptions of Print and Online Newspaper Journalists. Journalism and Mass Communication Quarterly, 82(2), 264-280. https://doi.org/10.1177/107769900508200203

Chaffee, S. \& Metzger, M. (2001). The end of mass communication. Mass Communications and Society, 4(4), 365-379. https://doi.org/10.1207/S15327825MCS0404_3

ComScore. (2019). Latest Rankings. https://www.comscore.com/lat/Prensa-y-Eventos/ Market-Rankings

Craft, S., Vos. T., \& Wolfgang, D. (2015). Reader Comments as Press Criticism: Implications for the Journalistic Field. Journalism, 17(6), 677-693. https://doi.org/10.1177/1464884915579332 
Cooperativa. (n.d.). La Compañía. Cooperativa.cl. https://www.cooperativa.cl/noticias/site/ edic/base/port/home_modulo_informacion_corporativa.html?1

Diario Financiero. (n.d.). Quiénes somos (Who are we?). df.cl. https://www.df.cl/noticias/quienes-somos/2018-09-20/114714.html

Domingo, D. (2008). Interactivity in the Daily Routines of Online Newsrooms: Dealing with an Uncomfortable Myth. Journal of Computer-Mediated Communication, 13(3), 680-704. https://doi.org/10.1111/j.1083-6101.2008.00415.x

Doval-Avendaño, M. \& Algarra, M. (2021). Periodismo y tecnología, tendencias de investigación y propuestas (Journalism and technology, research trends and proposals). Estudios sobre el mensaje periodístico, 27(2), 463-480. https://doi.org/10.5209/esmp.71073

Duffy, A. (2019). Out of the shadows: the editor as a defining characteristic of journalism. Journalism, 22 (3), 634-649. https://doi.org/10.1177/1464884919826818

Eisenhardt, K. (1989). Building theories from case study research. Academic Manage Review, 14(4), 532-550. https://doi.org/10.5465/amr.1989.4308385

El Dínamo. (n.d.). Política editorial (Editorial guidelines). Eldinamo.cl. https://www.eldinamo.cl/politica-editorial/

El Líbero. (n.d.). Quiénes somos (Who are we?). ellibero.cl. https://ellibero.cl/quienes-somos/

Emol. (n.d.). Términos y condiciones generales de uso (Use terms and conditions). Emol.com. https://www.emol.com/Terminos/Terminosycondiciones.aspx

El Mostrador (n.d.). Principios editoriales (Editorial line). https://media.elmostrador.cl/2010/11/PrincipiosEditoriales.pdf

Fontcuberta, M. (1999). Pauta y calidad informativa (Guidelines and information quality). Cuadernos.info, (13), 61-69. https://doi.org/10.7764/cdi.13.197

Fortunati, R. \& Sarrica, M. (2011). Insights from journalists on the future of the press. Communications, 36(2), 123-146. https://doi.org/10.1515/comm.2011.007

Gans, H. (1980). Deciding What's News: A Study of CBS Evening News, NBC Nightly News, Newsweek and Time. Vintage.

Gans, H. (2003). Democracy and the News. Oxford University Press.

Greene González, F. (2016). Medios online chilenos: diferencias en algunas prácticas periodísticas respecto de los medios escritos tradicionales (Chilean online media: differences in some journalistic practices over traditional print media). Revista de Comunicación, 15, 34-47.

Greene González, F. (2017). Algunas influencias en la autonomía de los periodistas chilenos: una actualización de investigaciones previas (Some influences on the autonomy of Chilean journalists: an update of previous research). Revista de Comunicación, 16(2), 234-251. https://doi.org/10.26441/RC16.2-2017-A11

Greene-González, M. F. \& Lecaros-Menéndez, M. J. (2015). El trabajo del editor y la pauta en medios online chilenos (The Work of the Editor and the Norms for Chilean Online Media). Palabra Clave, 18(2), 588-613. https://doi.org/10.5294/pacla.2015.18.2.11 
Gronemeyer, M. E. (2013). La digitalización y sus efectos sobre las prácticas y productos periodísticos en Chile (Digitalization and its Effect on Journalism Products and Practices in Chile). Palabra Clave, 16(1), 101-128. https://doi.org/10.5294/pacla.2013.16.1.4

Gunther, A. C. \& Liebhart, J. L. (2006). Broad reach or biased source? Decomposing the hostile media effect. Journal of Communication, 56(3), 449-466. https://doi.org/10.1111/j.1460-2466.2006.00295.x

Hamilton, J. (2004). All the news that's fit to sell. Princeton University Press.

Henkel, I., Thurman, N., Möller, J., \& Trilling, D. (2020). Do Online, Offline, and Multiplatform Journalists Differ in their Professional Principles and Practices? Findings from a Multinational Study.Journalism Studies, 21(19), 1363-1383. https://doi.org/10.1080/1461670X.2020.1749111

Hermida, A. (2011). Tweet the news: Social media streams and the practice of journalism. In S. Allan (Ed.), The Routledge companion to news and journalism (2nd ed.) (pp. 671-82). Routledge.

Hanusch, F. (2017). Web Analytics and the Functional Differentiation of Journalism Cultures: Individual, Organizational and Platform-Specific Influences on Newswork. Information, Communication and Society, 20(10), 1571-1586. https://doi.org/10.1080/1369118X.2016.1241294

Jofré, C. (2017). Journalism and news cultures: journalistic practices and online media in the Chilean newsroom. (Doctoral Dissertation, Monash University) https://doi.org/10.4225/03/58b76e231b545

Klinenberg, E. (2005). Convergence: News Production in a Digital Age. Annals of the American Academy of Political and Social Science, 597(1) 48-64.

Krippendorff, K. (2004). Content analysis: An introduction to its methodology (2nd ed.). Sage.

La Tercera. (n.d.). Términos y condiciones (Use terms and conditions). Latercera.cl. https://www.latercera.com/terminos-y-condiciones/

Lecaros, M. \& Greene, F. (2012). Editores chilenos y rutinas periodísticas: revisión y propuesta para un estudio sobre el cambio en la sala de redacción (Chilean Editors and Journalistic Routines: Review and Proposal Towards a Study on Change in Newsrooms). Cuadernos.info, (30), 53-60. https://doi.org/10.7764/cdi.30.426

Lee, C. S. \& Ma, L. (2012). News sharing in social media: the effect of gratifications and prior experience. Computers in Human Behavior, 28(2), 331-339. https://doi.org/10.1016/j.chb.2011.10.002

Lee, A., Lewis S., \& Powers, M. (2014) Audience clicks and news placement: A study of timelagged influence in online journalism. Communication Research, 41(4), 505-530. https://doi.org/10.1177/0093650212467031

Lee, E. \& Tandoc, E. (2017). When News Meets the Audience: How Audience Feedback Online Affects News Production and Consumption. Human Communication Research, 43(4), 436-449. https://doi.org/10.1111/hcre.12123

Lewis, S. \& Usher, N. (2013). Open Source and Journalism: Toward New Frameworks for Imagining News Innovation. Media, Culture and Society, 35(5), 602-619. https://doi.org/10.1177/0163443713485494

Lindlof, T. R. \& Taylor, B. C. (2011). Sensemaking: Qualitative Data Analysis and Interpretation. Qualitative Communication Research Methods, 3(1), 241-281. 
MacGregor, P. (2007) Tracking the online audience. Journalism Studies, 8(2), 280-298. https://doi.org/10.1080/14616700601148879

Mitchelstein, E. \& Boczkowski, P. (2009). Between tradition and change: A review of recent research on online news production. Journalism, 10(5), 562-586. https://doi. org/10.1177/1464884909106533

Navarrete, J. M. (2000). El muestreo en la investigación cualitativa (Sampling in qualitative research). Investigaciones sociales, 4(5), 165-180. https://doi.org/10.15381/is.v4i5.6851

O'Sullivan, J. \& Heinonen, A. (2008). Old values, new media: Journalism role perceptions in a changing world. Journalism Practice, 2(3), 357-371.https://doi.org/10.1080/17512780802281081

Pauta. (n.d.). Quiénes somos (Who are we?). pauta.cl. https://ellibero.cl/quienes-somos/

Pavlik, J. (2008). Media in the digital age. Columbia University Press.

Pellegrini, S. (1989). Políticas de Información (Information Policies). Ediciones Universidad Católica.

Puente, S. \& Grassau, D. (2011). Periodismo ciudadano: dos términos contradictorios. La experiencia chilena según sus protagonistas (Citizen Journalism: Two Contradictory Terms. The Chilean Experience as Their Own Players). Palabra Clave, 14(1), 137-155. https://doi.org/10.5294/pacla.2011.14.1.8

Puente, S., Edwards, C., \& Delpiano, M. O. (2014). Modelamiento de los aspectos intervinientes en el proceso de pauta periodística (Modeling the Aspects Intervening in Standard Journalistic Process). Palabra Clave, 17(1), 188-210. https://doi.org/10.5294/pacla.2014.17.1.7

Quandt, T. (2008). (No) news on the World Wide Web? Journalism Studies, 9(5), 717-738. https://doi.org/10.1080/14616700802207664

Ragas, M. W., Tran, H. L., \& Martin, J. A. (2014). Media-induced or search-driven? A study of online agenda-setting effects during the BP oil disaster. Journalism Studies, 15(1), 48-63. https://doi.org/10.1080/1461670X.2013.793509

Salaverría, R. (2016). Ciberperiodismo en Iberoamérica (Cyberjournalism in Iberoamerica). Fundación Telefónica y Ariel.

Saldaña, J. (2011). Fundamentals of qualitative research. Oxford University Press.

Salwen, M. (2005). Online news trends. In M. Salwen, B. Garrison, \& P. Driscoll (Eds.), Online News and the Public (pp. 47-77). Lawrence Erlbaum.

Shoemaker, P. \& Vos, T. (2009). Gatekeeping theory. Routledge.

Schudson, M. \& Anderson, C. (2009). Objectivity, Professionalism, and Truth Seeking in Journalism. In K. Wahl-Jorgensen \& T. Hanitzsch (Eds.), Handbook of Journalism Studies (pp. 88-101). Routledge.

Singer, J. (2004). More than Ink-stained Wretches: The Resocialization of Print Journalist in Converged Newsrooms. Journalism \& Mass Communication Quarterly, 81(4), 838-856. https://doi.org/10.1177/107769900408100408

Singer, J. (2011). Journalism and Digital Technologies. In W. Lowrey \& P. J Gade (Eds.), Changing the News: The Forces Shaping Journalism in Uncertain Times (pp. 213-229). Routledge. 
Singer, J., Hermida, A., Domingo, D., Heinonen, A., Paulussen, S., Quandt, T., Reich, Z., \& Vujnovic, M. (2011). Participatory Journalism: Guarding Open Gates at Online Newspapers. Wiley-Blackwell.

Stange, H. \& Salinas, C. (2009). Rutinas periodísticas. Discusión y trayectos teóricos sobre el concepto y su estudio en la prensa chilena (Journalistic routines. Discussion and theoretical trajectories on the concept and its study in the Chilean press). Cuadernos ICEI, 5, 1-41. https://www.researchgate.net/publication/333633385_Rutinas_periodisticas_ Discusion_y_trayectos_teoricos_sobre_el_concepto_y_su_estudio_en_la_prensa_chilena

Swanborn, P. (2008). Case-study's. Wat, Wanneer en Hoe? (Case Study. What, How and When?). Boom Onderwijs.

Tandoc, E. \& Vos, T. (2016). The journalist is marketing the news. Social media in the gatekeeping process. Journalism Practice, 10(8), 950-966. https://doi.org/10.1080/17512786.2015.1087811

Tandoc, E. \& Ferrucci. E. (2017). Giving in or Giving up: What Makes Journalists Use Audience, Feedback in Their News Work? Computers in Human Behavior, 68, 149-156. https://doi.org/10.1016/j.chb.2016.11.027

Tanner, E. (1999). Links to the World: the Internet in Chile, 1983-97. Gazette, 61, 39-57. https://doi.org/10.1177/0016549299061001003.

Tracy, S. J. (2019). Qualitative research methods: Collecting evidence, crafting analysis, communicating impact. John Wiley \& Sons.

TVN Corporativo. (2020). Historia (History). TVN.cl. https://www.tvn.cl/corporativo/ identificacionbasica/\#: :text=\%2D\%202001\%20Nace\%20el\%20portal\%20de,los\%20 m\%C3\%A1s\%20visitados\%20del\%20pa\%C3\%ADs

Vobič, I. (2015). From one-man band to integrated newsroom: Historicising online journalism developments in Slovenia. Journalism Studies, 16(2), 175-1. https://doi.org/10.1080/1461670X.2013.868143

$\mathrm{Vu}, \mathrm{H}$. (2014). The online audience as gatekeeper: The influence of reader metrics on news editorial selection. Journalism, 15(8), 1094-1110. https://doi.org/10.1177/1464884913504259

Weber, P. (2014). Discussions in the comments section: Factors influencing participation and interactivity in online newspapers' reader comments. New Media \& Society, 1(17), 941-957. https://doi.org/10.1177/1461444813495165

Welbers, K., Van Atteveldt, W., Kleinnijenhuis, J., Ruigrok, N., \& Schaper, J. (2016). News Selection Criteria in the Digital age: Professional Norms Versus Online Audience Metrics. Journalism, 17(8), 1037-1053. https://doi.org/10.1177/1464884915595474

Yin, R. K. (2009). Case Study Research: Design and Methods. Sage.

Zurstiege, G. (2006). The Reality of Media Research. Nordicom Review, 27(2), 85 - 92. https://www.nordicom.gu.se/sites/default/files/kapitel-pdf/242_zurstiege_1.pdf 


\section{SOBRE LAS AUTORAS}

FRANCISCA GREENE, es periodista de la Pontificia Universidad Católica de Chile y Doctora en Ciencias de la Información por la Universidad Complutense de Madrid. Es profesora en pregrado y en el Programa de Doctorado de la Facultad de Comunicación de la Universidad de los Andes. Sus líneas de investigación son rutinas y ética periodísticas.

(iD) https://orcid.org/0000-0002-0538-2584

MARÍA josÉ LECAROS, es periodista de la Pontificia Universidad Católica de Chile y Doctora en Opinión Pública por la Universidad de Navarra. Es presidenta del Consejo de Ética de la Federación de Medios de Chile y miembro del Consejo de Autorregulación Publicitaria, CONAR. Es profesora del Programa de Doctorado de la Facultad de Comunicación de la Universidad de los Andes. Sus investigaciones tratan sobre ética periodística.

(iD) https://orcid.org/ 0000-0001-5798-7860

FERNANDA CERDA, es periodista de la Universidad de los Andes y licenciada en Filosofía por esa misma universidad. Es Master en Comunicación Intercultural por la University of Sheffield, UK y Máster en Salud Pública con especialización en comunicación y salud por Boston University. Es profesora de la Facultad de Comunicación de la Universidad de los Andes. Actualmente está cursando un Doctorado en Comunicación en la misma casa de estudios.

iD https://orcid.org/ 0000-0001-5798-7860 